\title{
Approximate Noether gauge symmetries of the Bardeen model
}

\author{
U. Camci ${ }^{\mathrm{a}}$ \\ Department of Physics, Faculty of Science, Akdeniz University, 07058 Antalya, Turkey
}

Received: 29 October 2014 / Accepted: 21 November 2014 / Published online: 9 December 2014

(C) The Author(s) 2014. This article is published with open access at Springerlink.com

\begin{abstract}
We investigate the approximate Noether gauge symmetries of the geodesic Lagrangian for the Bardeen spacetime model. This is accommodated by a set of new approximate Noether gauge symmetry relations for the perturbed geodesic Lagrangian in the spacetime. A detailed analysis of the spacetime of the Bardeen model up to third-order approximate Noether gauge symmetries is presented.
\end{abstract}

\section{Introduction}

The black holes predicted in the framework of Einstein's gravity theory appear to exist in the Universe [1]. The existence of singularities inside the black holes was predicted by singularity theorems, and they indicate a breakdown of the classical theory [2]. A regular (i.e. non-singular) black hole which is characterized by the fact that the spacetime metric as well as the curvature invariants $R, R_{a b}, R^{a b}, R_{a b c d} R^{a b c d}$ do not present singularities anywhere was firstly derived from Einstein's field equations by Bardeen [3] which was initially approximately obtained. As shown in [4], the metric of the Bardeen model solves the Einstein equations with a self-gravitating nonlinear magnetic monopole as a source. The Bardeen model includes two parameters in the solution, namely the parameters $m$ and $e$. As usual for the Schwarzschild metric, the parameter $m$ can be interpreted as the mass parameter for the system. The other parameter $e$ can be interpreted as the magnetic charge of the geometry. Although the Bardeen model is always a regular black hole at $r=0$ for $e \neq 0$, it represents a regular black-hole spacetime only when the inequality $27 e^{2} \leq 16 \mathrm{~m}^{2}$ holds.

Let us briefly give some information as regards spacetime symmetries. A conformal Killing vector $(\mathrm{CKV}) \mathbf{K}$ has to satisfy $\mathfrak{f}_{\mathbf{K}} g_{a b}=2 \psi\left(x^{a}\right) g_{a b}$, where $g_{a b}$ is the metric tensor, $\mathfrak{f}_{\mathbf{K}}$ is the Lie derivative operator along $\mathbf{K}$, and $\psi\left(x^{a}\right)$ is a conformal factor. When $\psi_{; a b} \neq 0$, the CKV field is said to be

a e-mail: ucamci@akdeniz.edu.tr proper [5]. The vector field $\mathbf{K}$ is called the special conformal Killing vector (SCKV) field if $\psi_{; a b}=0$, the homothetic Killing vector (HKV) field if $\psi, a=0$, e.g. $\psi$ is a constant on the manifold, and the Killing vector (KV) field which gives the isometry if $\psi=0$. The set of all CKV (respectively SCKV, HKV, and KV) form a finite-dimensional Lie algebra. The maximum dimension of the CKV algebra on the manifold $M$ is 15 if $M$ is conformally flat, and it is seven if the spacetime is not conformally flat.

If a Lagrangian $L$ for a given dynamical system admits some symmetry, this property should strongly be related with Noether symmetries which describe physical features of differential equations possessing a Lagrangian $L$ in terms of first integrals admitted by them $[6,7]$. This can actually be seen in two ways. First of all, one can consider a strict Noether symmetry approach [8-10], which yields $£_{\mathbf{X}} L=0$, where $£_{\mathbf{X}}$ is the Lie derivative operator along $\mathbf{X}$. On the other side, one could use the Noether gauge symmetry (NGS) approach [1117], which is a generalization of the strict Noether symmetry approach in the sense that the Noether symmetry equation includes a gauge term in such a way that the vector field $\mathbf{X}=\xi \partial_{s}+\eta^{a} \frac{\partial}{\partial x^{a}}$ is called a NGS of a Lagrangian $L$ if there exists a gauge function, $A\left(s, x^{a}\right)$, such that the Noether symmetry condition holds $[6,7]$,

$\mathbf{X}^{[1]} L+L\left(D_{s} \xi\right)=D_{s} A$,

where $\mathbf{X}^{[\mathbf{1}]}=\mathbf{X}+\eta_{s}^{a} \frac{\partial}{\partial x^{a}}$ is the first prolongation operator of $\mathbf{X}, \eta_{s}^{a}=D_{s} \eta^{a}-\dot{x}^{a} D_{s} \xi$, and $D_{s}=\frac{\partial}{\partial s}+\dot{x}^{a} \frac{\partial}{\partial x^{a}}$ is the total derivative operator. The approximate symmetries are as useful as exact ones in a variety of problems arising from physics and applied mathematics. We will discuss how the approximate Noether gauge symmetries (ANGSs) of the perturbed geodesic Lagrangian are related to the geometrical symmetries of spacetimes in the following section.

The geodesic Lagrangian describing the motion of the massive or massless particles under the influence of a potential function $V\left(x^{c}\right)$ is 
$L\left(s, x^{c}, \dot{x}^{c}\right)=\frac{1}{2} g_{a b} \dot{x}^{a} \dot{x}^{b}-V\left(x^{c}\right)$,

where the dot represents the derivative with respect to the geodetic parameter $s$. The significance of an ANGS comes from the fact that a manifold having no exact symmetry can possess approximate ones. In this context, using the approximate symmetries of perturbed geodesic Lagrangian for spacetimes, one can recover the lost symmetries and conservation laws corresponding to the perturbed Lagrangian [18-22].

For the Schwarzschild metric, Kara et al. [20] have considered the approximate symmetries and the conservation laws of geodesic equations. Following the use of approximate symmetries for the Schwarzschild spacetime, Hussain et al. [21] have investigated the exact and approximate symmetries of the system of geodesic equations for the Reissner-Nordström (RN) spacetime. Using the ANGS for the Schwarzschild and Kerr metrics, Hussain et al. [22] have recovered all the lost conservation laws as trivial first-order approximate conservation laws in the first-order approximate case. Using approximate Lie symmetries the energy content of colliding plane waves have been studied by Sharif and Waheed [23]. By analyzing the behavior of the effective potential for the particles and photons, one has investigated the time-like and null geodesic structures for the Bardeen metric [24]. In a recent paper, Sharif and Waheed [25] have applied the approximate Lie symmetry method to the investigation of the energy re-scaling factor for Bardeen's regular black-hole solution. They only evaluated the third-order approximate symmetries of the orbital and geodesic equations using approximate Lie symmetry methods for the differential equations. Our main aim here is to extend our mathematical understanding of (1) by exploring its geometrical properties. We have obtained the geometrical nature of the ANGSs up to second-order perturbed Lagrangian and applied this method to the Schwarzschild and RN spacetimes [26]. It should be stressed that we deal with third-order ANGSs of the perturbed geodesic Lagrangian which have not been studied previously as far as we know.

The rest of the paper is organized as follows. In the following section, we present an analysis of third-order ANGSs of the first-order geodesic Lagrangian according to perturbed spacetime geometry. In Sect. 3, we apply the obtained results of Sect. 2 to the spacetime of the Bardeen model. Conclusions and discussions are presented in Sect. 4.

\section{Third-order approximate Noether gauge symmetries}

The calculation method of exact symmetries and the firstorder approximate symmetries of the Lagrangian are available in the literature [18-20]. Hussain et al. [21,22] have extended the procedure of calculating the approximate sym- metries of a perturbed Lagrangian to the second order. In this study we introduce a new geometrical method to calculate the ANGSs of the first-order perturbed Lagrangian extending the procedure of obtaining ANGSs until the third order.

The third-order ANGSs of the first-order perturbed Lagrangian,

$$
\begin{aligned}
& L\left(s, x^{a}, \dot{x}^{a}, \varepsilon\right)=L_{0}\left(s, x^{a}, \dot{x}^{a}\right)+\varepsilon L_{1}\left(s, x^{a}, \dot{x}^{a}\right) \\
& \quad+\varepsilon^{2} L_{2}\left(s, x^{a}, \dot{x}^{a}\right)+\varepsilon^{3} L_{3}\left(s, x^{a}, \dot{x}^{a}\right)+O\left(\varepsilon^{4}\right),
\end{aligned}
$$

is given by the ANGS generator

$\mathbf{X}=\mathbf{X}_{0}+\varepsilon \mathbf{X}_{1}+\varepsilon^{2} \mathbf{X}_{2}+\varepsilon^{3} \mathbf{X}_{3}$,

up to the gauge function $A\left(s, x^{a}\right)=A_{0}\left(s, x^{a}\right)+\varepsilon A_{1}\left(s, x^{a}\right)+$ $\varepsilon^{2} A_{2}\left(s, x^{a}\right)+\varepsilon^{3} A_{3}\left(s, x^{a}\right)$ if the ANGS generator satisfies the approximate Noether gauge symmetry conditions

$\mathbf{X}_{0}^{[1]} L_{0}+L_{0}\left(D_{s} \xi_{0}\right)=D_{s} A_{0}$,

$\mathbf{X}_{1}^{[1]} L_{0}+\mathbf{X}_{0}^{[1]} L_{1}+L_{0}\left(D_{s} \xi_{1}\right)+L_{1}\left(D_{s} \xi_{0}\right)=D_{s} A_{1}$,

$\mathbf{X}_{2}^{[1]} L_{0}+\mathbf{X}_{1}^{[1]} L_{1}+\mathbf{X}_{0}^{[1]} L_{2}+L_{0}\left(D_{s} \xi_{2}\right)+L_{1}\left(D_{s} \xi_{1}\right)$

$+L_{2}\left(D_{s} \xi_{0}\right)=D_{s} A_{2}$,

$\mathbf{X}_{3}^{[1]} L_{0}+\mathbf{X}_{2}^{[1]} L_{1}+\mathbf{X}_{1}^{[1]} L_{2}+\mathbf{X}_{0}^{[1]} L_{3}+L_{0}\left(D_{s} \xi_{3}\right)$

$+L_{1}\left(D_{s} \xi_{2}\right)+L_{2}\left(D_{s} \xi_{1}\right)+L_{3}\left(D_{s} \xi_{0}\right)=D_{s} A_{3}$,

where $\mathbf{X}_{0}$ is the exact NGS generator, $\mathbf{X}_{1}, \mathbf{X}_{2}$, and $\mathbf{X}_{3}$ are the first-order, second-order, and third-order ANGS generators, respectively, which are defined as

$\mathbf{X}_{j}=\xi_{j} \frac{\partial}{\partial s}+\eta_{j}^{a} \frac{\partial}{\partial x^{a}}, \quad(j=0,1,2,3)$,

$\mathbf{X}_{j}^{[1]}=\mathbf{X}_{j}+\eta_{j(s)}^{a} \frac{\partial}{\partial \dot{x}^{a}}, \quad \eta_{j(s)}^{a}=D_{s} \eta_{j}^{a}-\dot{x}^{a} D_{s} \xi_{j}$.

The above perturbed Lagrangian (3) yields a third-order (in $\varepsilon$ ) perturbed system of ordinary differential equations (ODEs). If $\mathbf{X}_{j}$ are the ANGSs corresponding to the perturbed Lagrangians $L_{j}\left(s, x^{a}, \dot{x}^{a}\right)$, then

$$
\begin{aligned}
I_{0}= & \xi_{0} L_{0}+\left(\eta_{0}^{a}-\xi_{0} \dot{x}^{a}\right) \frac{\partial L_{0}}{\partial \dot{x}^{a}}-A_{0}, \\
I_{1}= & \xi_{0} L_{1}+\xi_{1} L_{0}+\left(\eta_{0}^{a}-\xi_{0} \dot{x}^{a}\right) \frac{\partial L_{1}}{\partial \dot{x}^{a}} \\
& +\left(\eta_{1}^{a}-\xi_{1} \dot{x}^{a}\right) \frac{\partial L_{0}}{\partial \dot{x}^{a}}-A_{1}, \\
I_{2}= & \xi_{0} L_{2}+\xi_{1} L_{1}+\xi_{2} L_{0}+\left(\eta_{0}^{a}-\xi_{0} \dot{x}^{a}\right) \frac{\partial L_{2}}{\partial \dot{x}^{a}} \\
& +\left(\eta_{1}^{a}-\xi_{1} \dot{x}^{a}\right) \frac{\partial L_{1}}{\partial \dot{x}^{a}}+\left(\eta_{2}^{a}-\xi_{2} \dot{x}^{a}\right) \frac{\partial L_{0}}{\partial \dot{x}^{a}}-A_{2}, \\
I_{3}= & \xi_{0} L_{3}+\xi_{1} L_{2}+\xi_{2} L_{1}+\xi_{3} L_{0}+\left(\eta_{0}^{a}-\xi_{0} \dot{x}^{a}\right) \frac{\partial L_{3}}{\partial \dot{x}^{a}} \\
& +\left(\eta_{1}^{a}-\xi_{1} \dot{x}^{a}\right) \frac{\partial L_{2}}{\partial \dot{x}^{a}}+\left(\eta_{2}^{a}-\xi_{2} \dot{x}^{a}\right) \frac{\partial L_{1}}{\partial \dot{x}^{a}} \\
& +\left(\eta_{3}^{a}-\xi_{3} \dot{x}^{a}\right) \frac{\partial L_{0}}{\partial \dot{x}^{a}}-A_{3}
\end{aligned}
$$


are the first integrals associated with ANGSs $\mathbf{X}_{j}(j=0,1,2$, $3)$.

The spacetime metric $g_{a b}$ can be decomposed as follows:

$$
g_{a b}=\gamma_{a b}+\varepsilon h_{a b}+\varepsilon^{2} \sigma_{a b}+\varepsilon^{3} k_{a b}
$$

which means by (2) and (3) that the exact and perturbed geodesic Lagrangians of motion have the form

$L_{0}\left(s, x^{a}, \dot{x}^{a}\right)=\frac{1}{2} \gamma_{a b} \dot{x}^{a} \dot{x}^{b}-V\left(x^{c}\right)$,

$L_{1}\left(s, x^{a}, \dot{x}^{a}\right)=\frac{1}{2} h_{a b} \dot{x}^{a} \dot{x}^{b}$,

$L_{2}\left(s, x^{a}, \dot{x}^{a}\right)=\frac{1}{2} \sigma_{a b} \dot{x}^{a} \dot{x}^{b}$,

$L_{3}\left(s, x^{a}, \dot{x}^{a}\right)=\frac{1}{2} k_{a b} \dot{x}^{a} \dot{x}^{b}$,

where $\gamma_{a b}, h_{a b}, \sigma_{a b}$, and $k_{a b}$ are the exact, the first-order, the second-order, and the third-order perturbed metrics, respectively. The metric $\gamma_{a b}$ should be non-degenerate [i.e., $\left.\operatorname{det}\left(\gamma_{a b}\right) \neq 0\right]$. But the other metrics $h_{a b}, \sigma_{a b}$, and $k_{a b}$ can be degenerate [i.e., $\operatorname{det}\left(h_{a b}\right)=0, \operatorname{det}\left(\sigma_{a b}\right)=0, \operatorname{det}\left(k_{a b}\right)=0$ ] or non-degenerate, and they represent the slight deviations from flat spacetime geometry if the metric $\gamma_{a b}$ represents the flat geometry.

For the ANGS conditions (5-8) of the perturbed geodesic Lagrangians (16-19) we obtain

$$
\begin{aligned}
& \mathbf{X}_{j}^{[1]} L_{0}=\left(\gamma_{a d} \eta_{j, s}^{d}\right) \dot{x}^{a}+\frac{1}{2}\left(£_{\eta_{\mathbf{j}}} \gamma_{a b}-2 \xi_{j, s} \gamma_{a b}\right) \dot{x}^{a} \dot{x}^{b} \\
& -\left(\gamma_{b d} \xi_{j, a}\right) \dot{x}^{a} \dot{x}^{b} \dot{x}^{d}-\eta_{j}^{d} V_{, d}, \\
& \mathbf{X}_{j}^{[1]} L_{1}=\left(h_{a d} \eta_{j, s}^{d}\right) \dot{x}^{a}+\frac{1}{2}\left(£_{\eta_{\mathbf{j}}} h_{a b}-2 \xi_{j, s} h_{a b}\right) \dot{x}^{a} \dot{x}^{b} \\
& -\left(h_{b d} \xi_{j, a}\right) \dot{x}^{a} \dot{x}^{b} \dot{x}^{d}, \\
& \mathbf{X}_{j}^{[1]} L_{2}=\left(\sigma_{a d} \eta_{j, s}^{d}\right) \dot{x}^{a}+\frac{1}{2}\left(£_{\eta_{\mathbf{j}}} \sigma_{a b}-2 \xi_{j, s} \sigma_{a b}\right) \dot{x}^{a} \dot{x}^{b} \\
& -\left(\sigma_{b d} \xi_{j, a}\right) \dot{x}^{a} \dot{x}^{b} \dot{x}^{d}, \\
& \mathbf{X}_{j}^{[1]} L_{3}=\left(k_{a d} \eta_{j, s}^{d}\right) \dot{x}^{a}+\frac{1}{2}\left(£_{\eta_{\mathbf{j}}} k_{a b}-2 \xi_{j, s} k_{a b}\right) \dot{x}^{a} \dot{x}^{b} \\
& -\left(k_{b d} \xi_{j, a}\right) \dot{x}^{a} \dot{x}^{b} \dot{x}^{d},
\end{aligned}
$$

where $£_{\eta_{\mathbf{0}}}, £_{\eta_{1}}, £_{\eta_{2}}$, and $£_{\eta_{3}}$ are the geometrical derivative, i.e. the Lie derivative, the operators along $\eta_{\mathbf{0}}=\eta_{0}^{a} \partial_{x^{a}}, \eta_{\mathbf{1}}=$ $\eta_{1}^{a} \partial_{x^{a}}, \eta_{2}=\eta_{2}^{a} \partial_{x^{a}}$, and $\eta_{3}=\eta_{3}^{a} \partial_{x^{a}}$, respectively. Putting the above expressions into (5-8) together with $D_{s} \xi_{j}=\xi_{j, s}+$ $\xi_{j, d} \dot{x}^{d}$ and $D_{s} A_{j}=A_{j, s}+A_{j, d} \dot{x}^{d},(j=0,1,2,3)$, we find the ANGS conditions for (5):

$$
\begin{aligned}
& \xi_{0, a}=0, \gamma_{a b} \eta_{0, s}^{b}=A_{0, a} \\
& £_{\eta_{\mathbf{0}}} \gamma_{a b}=\xi_{0, s} \gamma_{a b} \\
& £_{\eta_{\mathbf{0}}} V=-\xi_{0, s} V-A_{0, s},
\end{aligned}
$$

for (6):

$\xi_{1, a}=0, \quad \gamma_{a b} \eta_{1, s}^{b}+h_{a b} \eta_{0, s}^{b}=A_{1, a}$

$£_{\eta_{\mathbf{1}}} \gamma_{a b}+£_{\eta_{\mathbf{0}}} h_{a b}=\xi_{1, s} \gamma_{a b}+\xi_{0, s} h_{a b}$

$£_{\eta_{\mathbf{1}}} V=-\xi_{1, s} V-A_{1, s}$,

for (7):

$\xi_{2, a}=0, \quad \gamma_{a b} \eta_{2, s}^{b}+h_{a b} \eta_{1, s}^{b}+\sigma_{a b} \eta_{0, s}^{b}=A_{2, a}$

$£_{\eta_{2}} \gamma_{a b}+£_{\eta_{1}} h_{a b}+£_{\eta_{\mathbf{0}}} \sigma_{a b}$

$$
=\xi_{2, s} \gamma_{a b}+\xi_{1, s} h_{a b}+\xi_{0, s} \sigma_{a b}
$$

$£_{\eta_{2}} V=-\xi_{2, s} V-A_{2, s}$,

and for (8):

$$
\begin{aligned}
& \xi_{3, a}=0, \gamma_{a b} \eta_{3, s}^{b}+h_{a b} \eta_{2, s}^{b}+\sigma_{a b} \eta_{1, s}^{b}+k_{a b} \eta_{0, s}^{b}=A_{3, a} \\
& \mathfrak{\eta}_{\eta_{3}} \gamma_{a b}+£_{\eta_{\mathbf{2}}} h_{a b}+£_{\eta_{\mathbf{1}}} \sigma_{a b}+£_{\eta_{\mathbf{0}}} k_{a b} \\
& =\xi_{3, s} \gamma_{a b}+\xi_{2, s} h_{a b}+\xi_{1, s} \sigma_{a b}+\xi_{0, s} k_{a b} \\
& \mathfrak{£}_{\eta_{\mathbf{3}}} V=-\xi_{3, s} V-A_{3, s} \text {. }
\end{aligned}
$$

Thus we find the geometrical form of the ANGS equations in terms of the Lie derivatives of exact and perturbed metrics up to the third order. Equations (24-26) are, of course, the conditions used to determine NGSs of exact geodesic Lagrangian. We have shown that the exact (5), the first-order (6), the second-order (7), and the third-order (8) form of the ANGS conditions are equivalent to the sets of equations (2426), (27-29), (30-32), and (33-35), respectively.

\section{Application to Bardeen model}

The Bardeen model describes a singularity-free black-hole spacetime, which means it is a regular spacetime. In this section, we will apply the above geometrical method to the third-order perturbed spacetime of the Bardeen model for finding the ANGSs of the perturbed geodesic Lagrangian. The line element representing the Bardeen spacetime is given by

$$
\begin{aligned}
\mathrm{d} s^{2}= & {\left[1-\frac{2 m r^{2}}{\left(r^{2}+e^{2}\right)^{3 / 2}}\right] \mathrm{d} t^{2}-\left[1-\frac{2 m r^{2}}{\left(r^{2}+e^{2}\right)^{3 / 2}}\right]^{-1} \mathrm{~d} r^{2} } \\
& -r^{2}\left(\mathrm{~d} \theta^{2}+\sin ^{2} \theta \mathrm{d} \phi^{2}\right)
\end{aligned}
$$

where $m$ is the mass of configuration and $e$ represents the magnetic charge of the nonlinear self-gravitating monopole, and we have taken the gravitational units, i.e. $G=c=1$. The Bardeen black-hole metric reduces to the Schwarzschild metric for $e=0$ and is flat for $m=0$. Using the expansions

$g_{t t}=1-\frac{\varepsilon}{r}+\frac{3 k \varepsilon^{3}}{2 r^{3}}+O\left(\varepsilon^{4}\right)$ 
and

$g_{r r}=-\left[1+\frac{\varepsilon}{r}+\frac{\varepsilon^{2}}{r^{2}}+\left(1-\frac{3 k}{2}\right) \frac{\varepsilon^{3}}{r^{3}}\right]+O\left(\varepsilon^{4}\right)$,

the first-order perturbed geodesic Lagrangian of the Bardeen model is given by

$$
\begin{aligned}
L= & \frac{1}{2}\left[\dot{t}^{2}-\dot{r}^{2}-r^{2}\left(\dot{\theta}^{2}+\sin ^{2} \theta \dot{\phi}^{2}\right)\right]-\frac{\varepsilon}{2 r}\left(\dot{t}^{2}+\dot{r}^{2}\right) \\
& -\frac{\varepsilon^{2}}{2 r^{2}} \dot{r}^{2}+\frac{\varepsilon^{3}}{2 r^{3}}\left[\frac{3 k}{2} \dot{t}^{2}-\left(1-\frac{3 k}{2}\right) \dot{r}^{2}\right] \\
& -V(t, r, \theta, \phi)+O\left(\varepsilon^{4}\right),
\end{aligned}
$$

where we take $\varepsilon=2 m$ and $e^{2}=k \varepsilon^{2}, 0<k \leq 4 / 27$, in the limit of a small mass of a gravitating point source. The above Lagrangian (39) yields

$$
\begin{aligned}
& L_{0}=\frac{1}{2}\left[\dot{t}^{2}-\dot{r}^{2}-r^{2}\left(\dot{\theta}^{2}+\sin ^{2} \theta \dot{\phi}^{2}\right)\right], \\
& L_{1}=-\frac{1}{2 r}\left(\dot{t}^{2}+\dot{r}^{2}\right), \\
& L_{2}=-\frac{1}{2 r^{2}} \dot{r}^{2} \\
& L_{3}=\frac{1}{2 r^{3}}\left[\frac{3 k}{2} \dot{t}^{2}-\left(1-\frac{3 k}{2}\right) \dot{r}^{2}\right],
\end{aligned}
$$

where we used $\gamma_{a b}=\operatorname{diag}\left(1,-1,-r^{2},-r^{2} \sin ^{2} \theta\right)$ which is the Minkowski metric, $h_{a b}=\operatorname{diag}(-1 / r,-1 / r, 0,0), \sigma_{a b}=$ $\operatorname{diag}\left(-1 / r^{2}, 0,0,0\right)$, and $k_{a b}=\operatorname{diag}\left(3 k / 2 r^{3},(3 k / 2-\right.$ $\left.1) / r^{3}, 0,0\right)$. Also, the Lagrangian (39) reduces to the geodesic Lagrangian of the Minkowski metric in the limit $\varepsilon \rightarrow 0$. Thus, we can apply the new set of the ANGSs obtained in the previous section to these exact metric $\gamma_{a b}$ and perturbed metrics $h_{a b}, \sigma_{a b}$, and $k_{a b}$ for the Bardeen spacetime. For the metric $\gamma_{a b}$, we find from the exact ANGS equations (24-26) the following 19 equations:

$\xi_{0, t}=0, \quad \xi_{0, r}=0, \quad \xi_{0, \theta}=0, \quad \xi_{0, \phi}=0$,

$\eta_{0, s}^{0}-A_{0, t}=0, \eta_{0, s}^{1}+A_{0, r}=0, r^{2} \eta_{0, s}^{2}+A_{0, \theta}=0$,

$r^{2} \sin ^{2} \theta \eta_{0, s}^{3}+A_{0, \phi}=0, \quad 2 \eta_{0, t}^{0}-\xi_{0, s}=0$,

$2 \eta_{0, r}^{1}-\xi_{0, s}=0, \quad 2 \eta_{0, \theta}^{2}+\frac{2}{r} \eta_{0}^{1}-\xi_{0, s}=0$,

$\frac{2}{r} \eta_{0}^{1}+2 \cot \theta \eta_{0}^{2}+2 \eta_{0, \phi}^{3}-\xi_{0, s}=0$,

$\eta_{0, r}^{0}-\eta_{0, t}^{1}=0, \quad \eta_{0, \theta}^{0}-r^{2} \eta_{0, t}^{2}=0$,

$\eta_{0, \phi}^{0}-r^{2} \sin ^{2} \theta \eta_{0, t}^{3}=0, \eta_{0, \theta}^{1}+r^{2} \eta_{0, r}^{2}=0$,

$\eta_{0, \phi}^{1}+r^{2} \sin ^{2} \theta \eta_{0, r}^{3}=0, \eta_{0, \phi}^{2}+\sin ^{2} \theta \eta_{0, \theta}^{3}=0$,

$V_{, t} \eta_{0}^{0}+V_{, r} \eta_{0}^{1}+V_{, \theta} \eta_{0}^{2}+V_{, \phi} \eta_{0}^{3}+V \xi_{0, s}+A_{0, s}=0$.
Considering both $\gamma_{a b}$ and $h_{a b}$, the first-order ANGS equations (27-29) yield again 19 equations:

$\xi_{1, t}=0, \quad \xi_{1, r}=0, \quad \xi_{1, \theta}=0, \quad \xi_{1, \phi}=0$,

$\eta_{1, s}^{0}-\frac{1}{r} \eta_{0, s}^{1}-A_{1, t}=0, \eta_{1, s}^{1}+\frac{1}{r} \eta_{0, s}^{1}+A_{1, r}=0$,

$r^{2} \eta_{1, s}^{2}+A_{1, \theta}=0, r^{2} \sin ^{2} \theta \eta_{1, s}^{3}+A_{1, \phi}=0$,

$\frac{1}{r} \eta_{0}^{1}-2 \eta_{0, t}^{0}+2 r \eta_{1, t}^{0}+\xi_{0, s}-r \xi_{1, s}=0$,

$\frac{1}{r} \eta_{0}^{1}-2 \eta_{0, r}^{1}-2 r \eta_{1, r}^{1}+\xi_{0, s}+\xi_{1, s}=0$,

$\frac{2}{r} \eta_{1}^{1}+2 \cot \theta \eta_{1}^{2}+2 \eta_{1, \phi}^{3}-\xi_{1, s}=0$,

$\frac{2}{r} \eta_{1}^{1}+\eta_{1, \theta}^{2}-\xi_{1, s}=0, \eta_{1, r}^{0}-\frac{1}{r}\left(\eta_{0, r}^{0}+\eta_{0, t}^{1}\right)-\eta_{1, t}^{1}=0$,

$\eta_{1, \theta}^{0}-\frac{1}{r} \eta_{0, \theta}^{0}-r^{2} \eta_{1, t}^{2}=0, \quad \eta_{1, \theta}^{1}+\frac{1}{r} \eta_{0, \theta}^{1}+r^{2} \eta_{1, r}^{2}=0$,

$\eta_{1, \phi}^{0}-\frac{1}{r} \eta_{0, \phi}^{0}-r^{2} \sin ^{2} \theta \eta_{1, t}^{3}=0$,

$\eta_{1, \phi}^{1}+\frac{1}{r} \eta_{0, \phi}^{1}+r^{2} \sin ^{2} \theta \eta_{1, r}^{3}=0$,

$\eta_{1, \phi}^{2}+\sin ^{2} \theta \eta_{1, \theta}^{3}=0$,

$V_{, t} \eta_{1}^{0}+V_{, r} \eta_{1}^{1}+V_{, \theta} \eta_{1}^{2}+V_{, \phi} \eta_{1}^{3}+V \xi_{1, s}+A_{1, s}=0$.

Using the metrics $\gamma_{a b}, h_{a b}$, and $\sigma_{a b}$, the second-order ANGS equations (30-32) give the following equations:

$\xi_{2, t}=0, \quad \xi_{2, r}=0, \quad \xi_{2, \theta}=0, \quad \xi_{2, \phi}=0$,

$\frac{1}{r} \eta_{1, s}^{0}-\eta_{2, s}^{0}+A_{2, t}=0$,

$\frac{1}{r^{2}}\left(r \eta_{1, s}^{1}+\eta_{0, s}^{1}\right)+\eta_{2, s}^{1}+A_{2, r}=0$,

$r^{2} \eta_{2, s}^{2}+A_{2, \theta}=0, r^{2} \sin ^{2} \theta \eta_{2, s}^{3}+A_{2, \phi}=0$

$\frac{1}{r^{2}}\left(\eta_{1}^{1}-2 r \eta_{1, t}^{0}+r \xi_{1, s}\right)+2 \eta_{2, t}^{0}-\xi_{2, s}=0$,

$\frac{1}{r^{3}}\left(2 \eta_{0}^{1}+r \eta_{1}^{1}-2 r^{2} \eta_{1, r}^{1}-2 r^{2} \eta_{0, r}^{1}+r \xi_{0, s}+r^{2} \xi_{1, s}\right)$

$-2 \eta_{2, r}^{1}+\xi_{2, s}=0, \quad \frac{2}{r} \eta_{2}^{1}+2 \eta_{2, \theta}^{2}-\xi_{2, s}=0$,

$\frac{2}{r} \eta_{2}^{1}+2 \cot \theta \eta_{2}^{2}+2 \eta_{2, \phi}^{3}-\xi_{2, s}=0, \eta_{2, \phi}^{2}+\sin ^{2} \theta \eta_{2, \phi}^{3}=0$,

$\eta_{2, \theta}^{0}-r^{2} \eta_{2, t}^{2}-\frac{1}{r} \eta_{1, \theta}^{0}=0, \quad \eta_{2, \phi}^{0}-r^{2} \sin ^{2} \theta \eta_{2, t}^{3}-\frac{1}{r} \eta_{1, \phi}^{0}=0$,

$\eta_{2, \theta}^{1}+r^{2} \eta_{2, r}^{2}+\frac{1}{r} \eta_{1, \theta}^{1}+\frac{1}{r^{2}} \eta_{0, \theta}^{1}=0$,

$\eta_{2, \phi}^{1}+r^{2} \sin ^{2} \theta \eta_{2, r}^{3}+\frac{1}{r} \eta_{1, \phi}^{1}+\frac{1}{r^{2}} \eta_{0, \phi}^{1}=0$,

$V_{, t} \eta_{2}^{0}+V_{, r} \eta_{2}^{1}+V_{, \theta} \eta_{2}^{2}+V_{, \phi} \eta_{2}^{3}+V \xi_{2, s}+A_{2, s}=0$.

Finally, the third-order ANGS equations (33-35) of the metrics $\gamma_{a b}, h_{a b}, \sigma_{a b}$, and $k_{a b}$ are explicitly written as 


$$
\begin{aligned}
& \xi_{3, t}=0, \quad \xi_{3, r}=0, \quad \xi_{3, \theta}=0, \quad \xi_{3, \phi}=0, \\
& \eta_{3, s}^{0}-\frac{1}{r} \eta_{2, s}^{0} \frac{3 k}{2 r^{3}} \eta_{0, s}^{0}-A_{3, t}=0, \\
& \frac{1}{r^{3}}\left[\left(1-\frac{3 k}{2}\right) \eta_{0, s}^{1}+r \eta_{1, s}^{1}+r^{2} \eta_{2, s}^{1}\right]+\eta_{3, s}^{1}+A_{3, r}=0, \\
& r^{2} \eta_{3, s}^{2}+A_{3, \theta}=0, r^{2} \sin ^{2} \theta \eta_{3, s}^{3}+A_{3, \phi}=0, \\
& 2 \eta_{3, t}^{0}+\frac{1}{r^{2}}\left(\eta_{2}^{1}-2 r \eta_{2, t}^{0}+r \xi_{2, s}\right) \\
& -\frac{9 k}{2 r^{4}} \eta_{0}^{1}+\frac{3 k}{2 r^{3}}\left(2 \eta_{0, t}^{0}-\xi_{0, s}\right)-\xi_{3, s}=0, \\
& 2 \eta_{1}^{1}+r \eta_{2}^{1}-2 r^{2} \eta_{2, r}^{1}-2 r \eta_{1, r}^{1}+r \xi_{1, s}+r^{2} \xi_{2, s}-\frac{3 k}{2} \xi_{0, s} \\
& +\frac{3(3 k-2)}{2 r} \eta_{0}^{1}+(3 k-2) \eta_{0, r}^{1}+r^{3}\left(-2 \eta_{3, r}^{1}+\xi_{3, s}\right)=0, \\
& \frac{2}{r} \eta_{3}^{1}+2 \eta_{3, \theta}^{2}-\xi_{3, s}=0, \eta_{3, \phi}^{2}+\sin ^{2} \theta \eta_{3, \phi}^{3}=0, \\
& \frac{2}{r} \eta_{3}^{1}+2 \cot \theta \eta_{3}^{2}+2 \eta_{3, \phi}^{3}-\xi_{3, s}=0 \\
& \eta_{3, r}^{0}-\eta_{3, t}^{1}-\frac{1}{r}\left(\eta_{2, r}^{0}+\eta_{2, t}^{1}\right)-\frac{1}{r^{2}} \eta_{1, t}^{1} \\
& +\frac{3 k}{2 r^{3}}\left[\eta_{0, r}^{0}+(3 k-2) \eta_{0, t}^{1}\right]=0, \\
& \eta_{3, \theta}^{0}-r^{2} \eta_{3, t}^{2}-\frac{1}{r} \eta_{2, \theta}^{0}+\frac{3 k}{2 r^{3}} \eta_{0, \theta}^{0}=0, \\
& \eta_{3, \phi}^{0}-r^{2} \sin ^{2} \theta \eta_{3, t}^{3}-\frac{1}{r} \eta_{2, \phi}^{0}+\frac{3 k}{2 r^{3}} \eta_{0, \phi}^{0}=0, \\
& \eta_{3, \theta}^{1}+r^{2} \eta_{2, r}^{3}+\frac{1}{r} \eta_{2, \theta}^{1}+\frac{1}{r^{2}} \eta_{1, \theta}^{1}+\frac{(2-3 k)}{2 r^{3}} \eta_{0, \theta}^{1}=0, \\
& \eta_{3, \phi}^{1}+r^{2} \sin ^{2} \theta \eta_{3, r}^{3}+\frac{1}{r} \eta_{2, \phi}^{1}+\frac{1}{r^{2}} \eta_{1, \phi}^{1}+\frac{(2-3 k)}{2 r^{3}} \eta_{0, \phi}^{1}=0, \\
& V_{, t} \eta_{3}^{0}+V_{, r} \eta_{3}^{1}+V_{, \theta} \eta_{3}^{2}+V_{, \phi} \eta_{3}^{3}+V \xi_{3, s}+A_{3, s}=0 .
\end{aligned}
$$

We simultaneously solved the exact, the first-order, the second-order, and the third-order ANGS equations (44-47) for the constant potential, e.g. $V(t, r, \theta, \phi)=V_{0}$. From (4447), we find for the exact ANGSs

$$
\begin{gathered}
\xi_{0}=a_{1}, \eta_{0}^{0}=a_{2}, \eta_{0}^{1}=0, \eta_{0}^{2}=-a_{3} \cos \phi+a_{4} \sin \phi, \\
\eta_{0}^{3}=\cot \theta\left(a_{3} \sin \phi+a_{4} \cos \phi\right)+a_{5}, \quad A_{0}=a_{6},
\end{gathered}
$$

for the first-order ANGSs

$$
\xi_{1}=b_{1}, \eta_{1}^{0}=b_{2}, \eta_{1}^{1}=0, \eta_{1}^{2}=-b_{3} \cos \phi+b_{4} \sin \phi,
$$

$\eta_{1}^{3}=\cot \theta\left(b_{3} \sin \phi+b_{4} \cos \phi\right)+b_{5}, \quad A_{1}=b_{6}$,

for the second-order ANGSs

$$
\begin{aligned}
& \xi_{2}=c_{1}, \eta_{2}^{0}=c_{2}, \eta_{2}^{1}=0, \eta_{2}^{2}=-c_{3} \cos \phi+c_{4} \sin \phi, \\
& \eta_{2}^{3}=\cot \theta\left(c_{3} \sin \phi+c_{4} \cos \phi\right)+c_{5}, \quad A_{2}=c_{6},
\end{aligned}
$$

and for the third-order ANGSs

$$
\begin{aligned}
\xi_{3} & =d_{1} \frac{s^{2}}{2}+d_{2} s+d_{3}, \\
\eta_{3}^{0} & =\frac{s}{2}\left(d_{1} t+2 d_{4}\right)+d_{2} \frac{t}{2}+d_{5}, \\
\eta_{3}^{1} & =\frac{r}{2}\left(d_{1} s+d_{2}\right), \quad \eta_{3}^{2}=d_{6} \cos \phi+d_{7} \sin \phi, \\
\eta_{3}^{3} & =\cot \theta\left(d_{6} \cos \phi-d_{7} \sin \phi\right)+d_{8}, \\
A_{3} & =\frac{1}{4} d_{1}\left(t^{2}-r^{2}-2 V_{0} s^{2}\right)-d_{2} V_{0} s+d_{4} t+d_{9},
\end{aligned}
$$

where $a_{\ell}, b_{\ell}, c_{\ell}(\ell=1, \ldots, 6)$, and $d_{1}, \ldots, d_{9}$ are the arbitrary constants of integration associated with the ANGSs.

It is easily seen from the above solutions that we have five exact, first-order, and second-order ANGSs and eight third-order ANGSs, which includes also exact ones. It is well known that the spacetime of the Bardeen model has four KVs, i.e.,

$\mathbf{K}_{0}=\partial_{t}, \quad \mathbf{K}_{1}=\cos \phi \partial_{\theta}-\cot \theta \sin \phi \partial_{\phi}$,
$\mathbf{K}_{2}=\sin \phi \partial_{\theta}+\cot \theta \cos \phi \partial_{\phi}, \quad \mathbf{K}_{3}=\partial_{\phi}$,

which correspond to the conservation of energy and angular momentum only.

For all orders of the ANGSs, we point out here that there is the vector field $\mathbf{Y}_{0}=\partial_{s}$, which gives a translation in the geodetic parameter $s$ and it always exists for a geodesic Lagrangian of the type (2), and the remaining ones are the four KVs given in (59) and (60). We found that the exact, the first-order, and the second-order ANGS algebras admitted by the Bardeen model are five dimensional. In addition to those of five ANGSs, there are three additional third-order ANGSs, namely the following:

$\mathbf{Y}_{1}=s \mathbf{Y}_{0}$, with gauge term $A_{3}=t$,

$\mathbf{Y}_{2}=s \partial_{s}+\frac{1}{2}\left(t \partial_{t}+r \partial_{r}\right)$

with gauge term $A_{3}=-V_{0} s$,

$\mathbf{Y}_{3}=s\left(s \partial_{s}+t \partial_{t}+r \partial_{r}\right)$,

$$
\text { with gauge term } A_{3}=\frac{1}{2}\left(t^{2}-r^{2}-2 V_{0} s^{2}\right) \text {. }
$$

Thus, it is seen that for the Bardeen model the conservation laws of linear momentum and spin angular momentum in the third-order approximation are lost. In the third-order approximate symmetry case, we compute the ANGSs of the perturbed geodesic Lagrangian for the Bardeen metric with non-constant potential. In order to determine the ANGSs associated with a given non-constant potential $V(t, r, \theta, \phi)$, one has to use the new ANGS equations (44-47). We have collected our results in Table 1.

Now, we intend to recover the lost conservation laws. Therefore, we will consider the lower than third order, i.e. the second-order, approximate symmetry of the Bardeen model. Thus we will retain the second-order terms in $\varepsilon$ and neglect 
Table 1 Third-order approximate Noether gauge symmetries and associated gauge functions $A_{0}, A_{1}, A_{2}$, and $A_{3}$ of the Bardeen metric for some non-constant potentials $V(t, r, \theta, \phi)$. Here $V_{0}$ is a non-zero constant

\begin{tabular}{|c|c|c|c|c|c|}
\hline Potential & ANGSs & $A_{0}$ & $A_{1}$ & $A_{2}$ & $A_{3}$ \\
\hline \multirow[t]{2}{*}{$V=\frac{V_{0}}{r}$} & $\mathbf{K}_{0}, \mathbf{K}_{1}, \mathbf{K}_{2}, \mathbf{K}_{3}, \mathbf{Y}_{0}$ & 0 & 0 & 0 & 0 \\
\hline & $\mathbf{Y}_{1}$ & 0 & 0 & 0 & $t$ \\
\hline \multirow[t]{2}{*}{$V=\frac{V_{0}}{r^{2}}$} & $\mathbf{K}_{0}, \mathbf{K}_{1}, \mathbf{K}_{2}, \mathbf{K}_{3}, \mathbf{Y}_{0}, \mathbf{Y}_{2}$ & 0 & 0 & 0 & 0 \\
\hline & $\mathbf{Y}_{1}, \mathbf{Y}_{3}$ & 0 & 0 & 0 & $t, \frac{1}{2}\left(t^{2}-r^{2}\right)$ \\
\hline \multirow[t]{2}{*}{$V=\frac{V_{0}}{r \sin \theta}$} & $\mathbf{K}_{0}, \mathbf{K}_{3}, \mathbf{Y}_{0}$ & 0 & 0 & 0 & 0 \\
\hline & $\mathbf{Y}_{1}$ & 0 & 0 & 0 & $t$ \\
\hline \multirow{2}{*}{$V=\frac{V_{0}}{r^{2} \sin \theta}$} & $\mathbf{K}_{0}, \mathbf{K}_{3}, \mathbf{Y}_{0}, \mathbf{Y}_{2}$ & 0 & 0 & 0 & 0 \\
\hline & $\mathbf{Y}_{1}, \mathbf{Y}_{3}$ & 0 & 0 & 0 & $t, \frac{1}{2}\left(t^{2}-r^{2}\right)$ \\
\hline \multirow[t]{2}{*}{$V=V_{0} r^{2}$} & $\mathbf{K}_{0}, \mathbf{K}_{1}, \mathbf{K}_{2}, \mathbf{K}_{3}, \mathbf{Y}_{0}$ & 0 & 0 & 0 & 0 \\
\hline & $\mathbf{Y}_{1}$ & 0 & 0 & 0 & $t$ \\
\hline \multirow[t]{2}{*}{$V=V_{0} t^{2}$} & $\mathbf{K}_{1}, \mathbf{K}_{2}, \mathbf{K}_{3}, \mathbf{Y}_{0}$ & 0 & 0 & 0 & 0 \\
\hline & $\sin \left(\sqrt{2 V_{0}} s\right) \mathbf{K}_{0}, \cos \left(\sqrt{2 V_{0}} s\right) \mathbf{K}_{0}$ & 0 & 0 & 0 & $\begin{array}{l}\sqrt{2 V_{0}} \cos \left(\sqrt{2 V_{0}} s\right) \\
-\sqrt{2 V_{0}} \sin \left(\sqrt{2 V_{0}} s\right)\end{array}$ \\
\hline \multirow[t]{2}{*}{$V=\frac{V_{0}}{8}\left(r^{2}-t^{2}\right)$} & $\mathbf{K}_{1}, \mathbf{K}_{2}, \mathbf{K}_{3}, \mathbf{Y}_{0}$ & 0 & 0 & 0 & 0 \\
\hline & $\begin{array}{l}e^{V_{0} s}\left[\left(1-v_{0} s\right) \mathbf{Y}_{0}+V_{0} \mathbf{Y}_{2}\right] \\
e^{V_{0} s}\left[\left(1+v_{0} s\right) \mathbf{Y}_{0}-V_{0} \mathbf{Y}_{2}\right] \\
e^{V_{0} s / 2} \mathbf{Y}_{0}, e^{-V_{0} s / 2} \mathbf{Y}_{0}\end{array}$ & 0 & 0 & 0 & $\begin{array}{l}-\frac{V_{0}^{2}}{4}\left(t^{2}-r^{2}\right) e^{V_{0} s},-\frac{V_{0}^{2}}{4}\left(t^{2}-r^{2}\right) e^{-V_{0} s} \\
\quad-\frac{V_{0}}{2} t e^{-V_{0} s / 2}, \frac{V_{0}}{2} t e^{V_{0} s / 2}\end{array}$ \\
\hline \multirow[t]{2}{*}{$V=V_{0} e^{\lambda \phi}$} & $\mathbf{K}_{0}, \mathbf{Y}_{0}, \mathbf{Y}_{2}-\frac{1}{\lambda} \mathbf{K}_{3}(\lambda \neq 0)$ & 0 & 0 & 0 & 0 \\
\hline & $\mathbf{Y}_{1}$ & 0 & 0 & 0 & $t$ \\
\hline \multirow[t]{2}{*}{$V=V_{0} \phi$} & $\mathbf{K}_{0}, \mathbf{Y}_{0}$ & 0 & 0 & 0 & 0 \\
\hline & $\mathbf{K}_{3}, \mathbf{Y}_{1}$ & $-V_{0} s, 0$ & $-V_{0} s, 0$ & $-V_{0} s, 0$ & $-V_{0} s, t$ \\
\hline \multirow[t]{2}{*}{$V=V_{0} t$} & $\mathbf{K}_{1}, \mathbf{K}_{2}, \mathbf{K}_{3}, \mathbf{Y}_{0}$ & 0 & 0 & 0 & 0 \\
\hline & $\mathbf{K}_{0}, \quad \mathbf{Y}_{2}-\frac{3 V_{0} s^{2}}{4} \mathbf{Y}_{0}, \quad \mathbf{Y}_{3}-\frac{V_{0} s^{3}}{4} \mathbf{Y}_{0}$ & $-V_{0} s, 0,0$ & $-V_{0} s, 0,0$ & $-V_{0} s, 0,0$ & $\begin{aligned}-V_{0} s, & \frac{V_{0} s}{2}\left(\frac{s^{2}}{2}-3 t\right) \\
& \frac{1}{2}\left(t^{2}-r^{2}\right)+\frac{V_{0} s^{2}}{2}\left(\frac{V_{0} s^{2}}{4}-3 t\right)\end{aligned}$ \\
\hline
\end{tabular}

$O\left(\varepsilon^{3}\right)$ in the geodesic Lagrangian (39) for the Bardeen model. It is noted that the metric of the Bardeen model as the second-order perturbed spacetime reduces to the secondorder perturbed Schwarzschild solution. So we will use the new set of determining equations up to second order to check whether the conservation laws are lost or not. Solving the exact, first-order and second-order ANGS equations (44-46) for the constant potential the same components follow as given by (48-49) and (50-51) for the exact and first-order ANGSs, respectively. But for the second-order ANGSs we have the following components:

$$
\begin{aligned}
\xi_{2}= & c_{1} \frac{s^{2}}{2}+c_{2} s+c_{3}, \\
\eta_{2}^{0}= & \frac{t}{2}\left(c_{1} s+c_{2}\right)-r \sin \theta\left(c_{4} \cos \phi-c_{5} \sin \phi\right) \\
& -c_{6} r \cos \theta+c_{7} s+c_{8}, \\
\eta_{2}^{1}= & \frac{r}{2}\left(c_{1} s+c_{2}\right)+t \sin \theta\left(-c_{4} \cos \phi+c_{5} \sin \phi\right) \\
& -\cos \theta\left(c_{6} t+c_{9} s+c_{10}\right) \\
& -\sin \theta\left[s\left(c_{11} \sin \phi-c_{12} \cos \phi\right)\right. \\
& \left.+c_{13} \cos \phi-c_{14} \sin \phi\right],
\end{aligned}
$$

$$
\begin{aligned}
\eta_{2}^{2}= & \frac{\cos \theta}{r}\left[t\left(-c_{4} \cos \phi+c_{5} \sin \phi\right)\right. \\
& \left.-s\left(c_{11} \sin \phi+c_{12} \cos \phi\right)-c_{13} \cos \phi+c_{14} \sin \phi\right] \\
& +\frac{\sin \theta}{r}\left(c_{6} t+c_{9} s+c_{10}\right)-c_{15} \cos \phi+c_{16} \sin \phi, \\
\eta_{2}^{3}= & \frac{1}{r \sin \theta}\left[t\left(c_{4} \sin \phi+c_{5} \cos \phi\right)\right. \\
& \left.+s\left(-c_{11} \cos \phi+c_{12} \sin \phi\right)+c_{13} \sin \phi+c_{14} \cos \phi\right] \\
& +\cot \theta\left(c_{15} \sin \phi+c_{16} \cos \phi\right)+c_{17}, \\
A_{2}= & \frac{1}{4} c_{1}\left(t^{2}-r^{2}-2 V_{0} s^{2}\right)-c_{2} V_{0} s+c_{7} t+c_{9} r \cos \theta \\
& +r \sin \theta\left(c_{11} \sin \phi+c_{12} \cos \phi\right)+c_{18},
\end{aligned}
$$

where $c_{1}, \ldots, c_{18}$ are constants of integration. It is immediately seen from these solutions that the "lost" symmetries for the Bardeen metric have appeared in the second-order ANGS solutions, and the second-order ANGS generators are the four $\mathrm{KVs}(59-60)$ and $\mathbf{Y}_{0}, \ldots, \mathbf{Y}_{3}$, and $\mathbf{Z}_{1}, \ldots, \mathbf{Z}_{9}$ given by (7082 ) in the appendix, where for the second-order ANGSs of the Bardeen model, the gauge functions are second order, $\mathbf{Y}_{2}$ has a gauge function such as $A_{2}=-V_{0} s$, and the gauge function 
of $\mathbf{Y}_{3}$ is also of the form $A_{2}=\frac{1}{2}\left(t^{2}-r^{2}-2 V_{0} s^{2}\right)$. Hence, we have recovered 17 ANGSs in the second-order approximate case of the perturbed Lagrangian for the Bardeen model, as is expected. We conclude that the first-order ANGSs that have been recovered by the first-order perturbed Schwarzschild metric are the second-order ANGSs for the Bardeen metric, which is also equivalent to the second-order perturbed Schwarzschild metric. The ANGSs and corresponding gauge functions of the second-order perturbed Schwarzschild solution are also computed for some non-constant potentials, and the results of the calculations are the same as the ones shown in the Table 1 of [26], but there is only one difference: the gauge function $A_{1}$ of the first-order perturbation should be replaced by the gauge function $A_{2}$ of the second-order perturbation in Table 1 of [26].

\section{Conclusions and discussions}

In this paper, we studied the geometric nature of the ANGSs up to third order (in $\varepsilon$ ) for the geodesic Lagrangian of spacetimes. We showed that the computation of third-order ANGSs of the perturbed first-order geodesic Lagrangian (3) is reduced to the problem of finding solutions for the new geometrical set of exact differential conditions (24-26), the first-order differential conditions (27-29), the second-order differential conditions (30-32), and the third-order differential conditions (33-35), which involve the exact, first-order, second-order and third-order ANGSs and the potential function defining the dynamical system. For the spacetime of the Bardeen model, this new method of finding ANGSs enables one to get an ANGS with non-zero potential.

It is pointed out in [25] that one cannot recognize the parameter $e$ of the Bardeen spacetime as the Coulomb charge $Q$ of the RN spacetime. We note that the Bardeen model with magnetic charge $e$ is the analog of the RN spacetime with electric charge $Q$. For the Bardeen model, although there is no term of $g_{t t}$ quadratic in $\varepsilon, g_{r r}$ includes a quadratic term [see (37) and (38)]. Therefore, getting the lost symmetries, it is sufficient to calculate the ANGSs of the RN spacetime for the second-order term of the perturbed Lagrangian, but this may not true for the Bardeen model in which the calculation is needed of the ANGSs up to third-order term in the perturbed Lagrangian.

Applying our new method to the Bardeen model up to the third-order approximation, we found that the number of exact, first-order, and second-order ANGSs is five. The number of third-order ANGSs was found to be eight-not 17 as is expected. That is, in the third-order approximate symmetry case, the linear and spin angular momentum conservation laws for the Bardeen spacetime are lost. Retaining only terms of first and second order in the metric coefficients $g_{t t}$ and $g_{r r}$ given in (37) and (38), and neglecting $O\left(\varepsilon^{3}\right)$, it reduces to the second-order perturbed spacetime for the Schwarzschild solution. Thus, for the Bardeen model as the second-order perturbed spacetime, we reconsider our new ANGS equations to check whether those of the lost conservation laws can be recovered. Then we find that for the Bardeen model all the lost conservation laws are recovered in the secondorder approximation.

It would be interesting to analyze the other special spacetimes according to the ANGSs in which one can find the answer of the question "what is the order of the ANGSs to give all conservation laws?". This issue will be a subject of research in the future.

Acknowledgments This work was supported by Akdeniz University, Scientific Research Projects Unit (BAP).

Open Access This article is distributed under the terms of the Creative Commons Attribution License which permits any use, distribution, and reproduction in any medium, provided the original author(s) and the source are credited.

Funded by $\mathrm{SCOAP}^{3}$ / License Version CC BY 4.0.

\section{Appendix: ANGSs for Schwarzschild spacetime}

The number of the ANGS generators of the first-order (in $\varepsilon$ ) geodesic Lagrangian for the Schwarzschild spacetime with vanishing potential is 17 [22]: the four KVs given in (59) and (60), and

$\mathbf{Y}_{0}=\partial_{s}$,

$\mathbf{Y}_{1}=s \mathbf{Y}_{0}$, with gauge term $A_{1}=t$,

$\mathbf{Y}_{2}=s \partial_{s}+\frac{1}{2}\left(t \partial_{t}+r \partial_{r}\right)$,

$\mathbf{Y}_{3}=s\left(s \partial_{s}+t \partial_{t}+r \partial_{r}\right)$,

$$
\text { with gauge term } A_{1}=\frac{1}{2}\left(t^{2}-r^{2}\right) \text {, }
$$

$\mathbf{Z}_{1}=\sin \theta \cos \phi \partial_{r}+\frac{\cos \theta \cos \phi}{r} \partial_{\theta}-\frac{\csc \theta \sin \phi}{r} \partial_{\phi}$,

$\mathbf{Z}_{2}=\sin \theta \sin \phi \partial_{r}+\frac{\cos \theta \sin \phi}{r} \partial_{\theta}+\frac{\csc \theta \cos \phi}{r} \partial_{\phi}$,

$\mathbf{Z}_{3}=\cos \theta \partial_{r}-\frac{\sin \theta}{r} \partial_{\theta}$,

$\mathbf{Z}_{4}=r \sin \theta \cos \phi \partial_{t}+t \mathbf{Z}_{1}$,

$\mathbf{Z}_{5}=r \sin \theta \sin \phi \partial_{t}+t \mathbf{Z}_{2}$,

$\mathbf{Z}_{6}=r \cos \theta \partial_{t}+t \mathbf{Z}_{3}$,

$\mathbf{Z}_{7}=s \mathbf{Z}_{1}$, with gauge term $A_{1}=-r \sin \theta \cos \phi$,

$\mathbf{Z}_{8}=s \mathbf{Z}_{2}$, with gauge term $A_{1}=-r \sin \theta \sin \phi$,

$\mathbf{Z}_{9}=s \mathbf{Z}_{3}$, with gauge term $A_{1}=-r \sin \theta$,

where the generators $\mathbf{Z}_{1}, \mathbf{Z}_{2}, \mathbf{Z}_{3}$ provide the conservation of linear momentum, and $\mathbf{Z}_{4}, \mathbf{Z}_{5}$, and $\mathbf{Z}_{6}$ yield the conservation 
of spin angular momentum. Using the generator $\mathbf{Y}_{2}$ one can write $s=t^{2}$ or $s=r^{2}$ which gives rise to the interpretation of the four $\mathrm{KVs} \mathbf{K}_{0}, \mathbf{K}_{1}, \mathbf{K}_{2}, \mathbf{K}_{3}$, and the 11 generators $\mathbf{Y}_{0}, \mathbf{Y}_{1}, \mathbf{Z}_{1}, \ldots, \mathbf{Z}_{9}$ being $\mathrm{CKV}$ s with conformal factor $\psi=\left(c_{1} t^{2}+c_{2}\right) / 2$. It is a very well-known fact that the Lie algebra of the CKVs for a conformally flat spacetime is 15 dimensional.

\section{References}

1. L. Ferrarese, H. Ford, Space Sci. Rev. 116, 523 (2005)

2. S.W. Hawking, G.F.R. Ellis, The Large Scale Structure of Spacetime (Cambridge University Press, Cambridge, 1973)

3. J.M. Bardeen, in Proceedings of GR5, Tbilisi, U.S.S.R., p. 174 (1968)

4. E. Ayon-Beato, A. Garcia, Phys. Lett. B 493, 149 (2000)

5. G.H. Katzin, J. Levine, W.R. Davis, J. Math. Phys. 10, 617 (1969)

6. H. Stephani, Differential Equations: Their Solution using Symmetries (Cambridge University Press, Cambridge, 1989), p. 99

7. N.H. Ibragimov, CRC Handbook of Lie Group Analysis of Differential Equations: Symmetries, Exact Solutions and Conservation Laws (CRC Press, Boca Raton, 1994)
8. S. Capozziello, G. Lambiase, Gen. Relat. Gravit. 32, 673 (2000)

9. U. Camci, Y. Kucukakca, Phys. Rev. D 76, 084023 (2007)

10. Y. Kucukakca, U. Camci, I. Semiz, Gen. Relat. Gravit. 44, 1893 (2012)

11. T. Feroze, F.M. Mahomed, A. Qadir, Nonlinear Dyn. 45, 65 (2006)

12. M. Tsamparlis, A. Paliathanasis, Gen. Relat. Gravit. 42, 2957 (2010)

13. Y. Kucukakca, U. Camci, Astrophys. Space Sci. 338, 211 (2012)

14. I. Hussain, M. Jamil, F.M. Mahomed, Astrophys. Space Sci. 337, $373(2011)$

15. F. Ali, T. Feroze, Int. J. Theor. Phys. 52, 3329 (2013)

16. U. Camci, JCAP 07, 002 (2014)

17. U. Camci, A. Yildirim, Phys. Scr. 89, 084003 (2014)

18. T. Feroze, A.H. Kara, Int. J. Nonlinear Mech. 37, 275 (2002)

19. A.H. Bokhari, A.H. Kara, A.R. Kashif, F.D. Zaman, Int. J. Theor. Phys. 45, 1063 (2006)

20. A.H. Kara, F.M. Mahomed, A. Qadir, Nonlinear Dyn. 51, 183 (2008)

21. I. Hussain, F.M. Mahomed, A. Qadir, SIGMA 3, 115 (2007)

22. I. Hussain, F.M. Mahomed, A. Qadir, Gen. Relat. Gravit. 41, 2399 (2009)

23. M. Sharif, S. Waheed, Braz. J. Phys. 42, 219 (2012)

24. S. Zhou, J. Chen, Y. Wang, Int. J. Mod. Phys. D 21, 1250077 (2012)

25. M. Sharif, S. Waheed, Phys. Scr. 83, 015014 (2011)

26. U. Camci, Gen. Relat. Gravit. 46, 1824 (2014) 